\title{
Impact of SMEs' logistics consulting support policy on business performance
}

Seok-do Hong , Ph.D.student, Dept. Of Smart Convergence Consulting, Hansung University, 02876, Seoul, Korea,Ourjesus1234@naver.com

Yen-yoo You, Professor, Dept. Of Smart Convergence Consulting, Hansung University, 02876, Seoul, Korea, threey0818@hansung.ac.kr

*Corresponding Author

Abstract. Background/Objectives: Recently, small and medium-sized enterprises (SMEs) have de creased their sales and profits in a rapidly changing business environment. The government $h$ as promoted a logistics consulting support policy to bolster the business performance of SME S.

Methods/Statistical analysis: Logistics consulting aims to improve logistics business processes, l ogistics services, and logistics operation management, and improve the business performance $b$ y supporting accounting services.

In this process, the logistics consulting performance of 150 SMEs was investigated to analyze the business performance of SMEs.

Findings: Through an analysis of these aims, they seem to be effective in business performan ce. Improvement in business process is to standardize a consistent process of the process, bu siness order and business performance. And also, improvement in services will bring good lev el of information quality, interaction quality, order effectiveness, order accuracy, product status , timeliness and support system. To improve operations management will be helpful to cost 1 evel due to unit cost management of furnaces, planned logistics function through order mana gement, work disruption prevention due to single order, and work efficiency through procedur al improvement, which is analyzed to increase business performance by affecting the back. In addition, the accounting business support was analyzed that it makes improvements in their business performance which includes accounting, management analysis, cost management, and budget management. Improvements/Applications: The result shows that the logistics consulti ng support policy is an effective way to enhanced the self-sufficiency of the SME, which will be an opportunity for job creation and development of logistics services.

Keywords: SMEs, logistics, consulting, distribution, business performance

Received: 11.12.2020 Accepted: 05.01.2021 $\quad$ Published: 03.02.2021

\section{INTRODUCTION}

With $99.9 \%$ of Korean companies being SMEs with less than 300 employees, fostering specialized logistics companies is very important. Some problems are pointing out with the smallness of the business, low productivity, logistics system inefficiency, and limitations of the growth of third-party logistics companies due to the growth of second-party logistics companies. The scale of investment in facilities and equipment is also $5.7 \%$ which is compared to the top Korean companies. In addition, among business areas, the transportation sector is outsourced to other companies, and the storage and inventory management divisions are only performing the simple tasks. The labor productivity is also low because of the lack of professional. The separated 3PL logistics service market does not build a base for growth. Furthermore, logistics standardization, logistics coordination, and logistics automation are insufficient with the problem. [1] Logistics service is divided into three components including the element in pre-trade, element on-trading, element in after-trading. [2] With the regard to logistics indicator measurement, the subjective measurement, cost calculation and net profits are considered very difficult to objectively obtain an indicator measurement of a meaningful comparative measurement method between sample companies. It means that the concept of indicator measurement and developed research methods should be continued. The business performance of SMEs targeted what small and medium-sized logistics companies can achieve by improving their performance. According to the previous research, introduction of logistics information system for logistics companies has the potential to affect corporate performance. [3] In order to boost the growth and development of the logistics consulting industry, the logistics 
consulting industry will have to find a multi-dimensional way to improve quality of the service and user satisfaction. In particular, in order to increase the satisfaction level, and make impressive performance of the logistics consulting project, consultants need to have responsibility for professional knowledge and attitude, ability to perform reliable work, ability to understand and consideration customers, and effort to provide customized services that respond to customer needs. [5] Qualified consultants offer the suggestions for identifying and analyzing the management problems of the user organization with an objective attitude, furthermore, an expert said that"when a customer requests assistance in implementing these solutions it is advice to provide service. [6]. The area of service that provided by the consultant defined as management consulting which focuses on providing assistance for the decision makers. [7] Logistics management is the recording, summarizing of the business, and reporting the business activities through the logistics management system.

\section{Materials and Methods}

The study aims to analyze a small business engaged in the logistics field as a sample of SMEs to look into that the logistics consulting and logistics work support have an effect on business performance. The purpose of this study is to analyze whether logistics business shows further improvement in business performance or not. The subjects of the survey were small and medium-sized enterprises, and a total of 150 copies were collected and analyzed. The program of SPSS 20. Frequency analysis, Factor analysis and Reliability analysis were utilized as the analysis method. In the case of the statistical analysis, the hypothesis test is the Linear regression analysis which was conducted to derive the results of this study.

\section{2-1. Research model}

The study is based on the theoretical background small and medium-sized logistics consulting to analyze the effect of SMEs' logistics consulting support on business performance. The improvement of Logistics business process, Logistics service improvement, Logistics operation management improvement, etc. were used as independent variables, and business performance were used as the dependent variable, logistics business support was used as a controlling variable. The logistics business process was divided into Logistics work plan, Logistics work order, Improvement, Standardization and so on. The level of logistics service consists of Information quality, Interaction quality, Logistics order effectiveness, Order accuracy, Product status, Timeliness and Logistics support system, etc. Logistics operation management consists of the cost level improvement through logistics unit price management, Improvement of planned logistics function due to order management, Improved prevention of business confusion due to order management, Improvement in logistics work efficiency. In addition, the improvement in procedure, Logistics business performance is accuracy of delivery-based yield, shortened lead time, Logistics cost reduction, etc. were set up as the target of performance. As a control variable Logistics accounting support, Logistics management analysis, and Logistics cost management were selected for logistics budget management, and research model was presented to measure the business performance of the logistics business. [Figure 1].

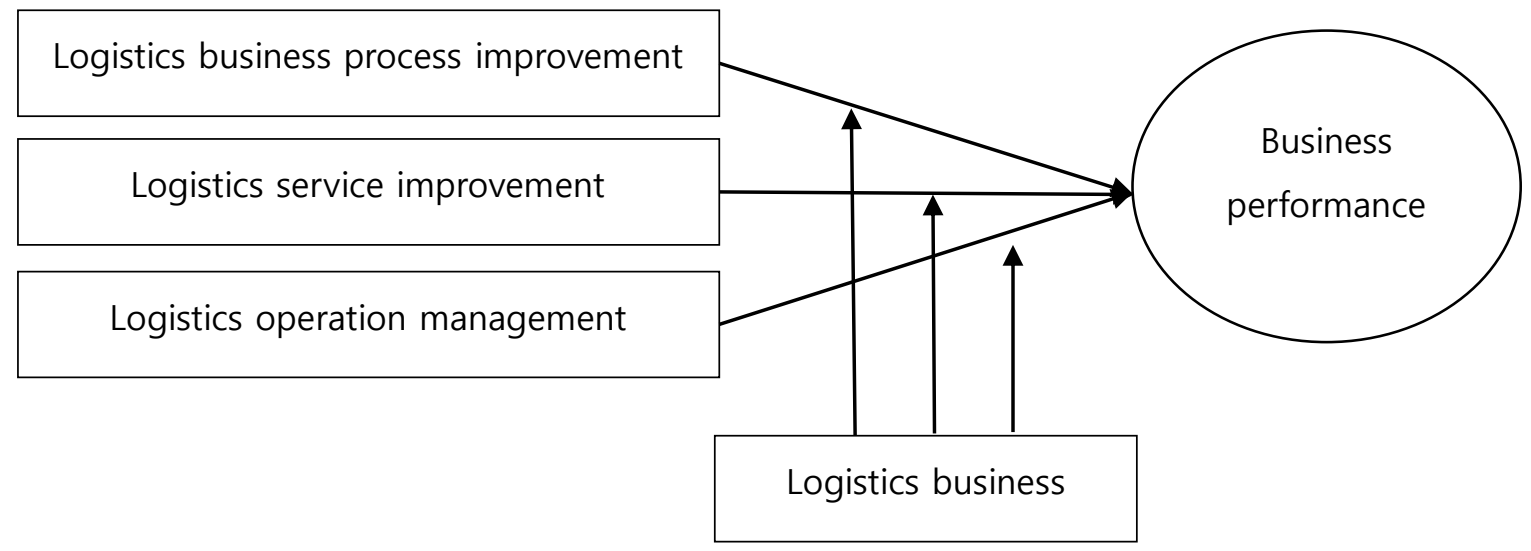

Figure 1. Research model 1

2-2. Research Hypothesis 
The hypothesis of this study conducted to manage the business performance of SMEs through logistics consulting, comprehensive diagnosis of logistics business management, logistics service management, logistics operation management, and logistics business support. It helps to enhance the competitiveness of companies. As supporting the project for small and medium-sized logistics companies, business improvement activities were conducted in their work processes, services, operations management and implementing policies of accounting operations to increase logistics performance. Based on the content above, the hypothesis is established as follows.

H1-1 Logistics business process improvement will have a positive (+) effect on business performance.

H1-2 Logistics service improvement will have a positive $(+)$ effect on business performance.

H1-3 Logistics business management improvement will have a positive (+) effect on business performance.

H2 Logistics business support will have a positive (+) effect on business performance.

H3-1 As the business process improvement affects business performance, accounting business support will act as a moderator.

H3-2 As the logistics service improvement affects business performance, accounting business support will act as a moderator.

H3-3 Logistics operation management improvement affects business performance, accounting business support will act as a moderator.

\section{2-3. Operational Definition of Variables}

In the study, the logistics consulting is used to understand the business performance of SMEs, and the operational definition was utilized to analyze that the logistics business support will have an impact on business performance. [Table 1].

Table 1. Operational definition of variables

\begin{tabular}{|c|c|c|c|}
\hline \multicolumn{2}{|l|}{ Variable } & Variable definition & $\begin{array}{l}\text { Prior } \\
\text { research }\end{array}$ \\
\hline \multirow{3}{*}{$\begin{array}{l}\text { SMEs } \\
\text { Logistics } \\
\text { consulting }\end{array}$} & $\begin{array}{l}\text { Logistics business } \\
\text { process } \\
\text { improvement }\end{array}$ & $\begin{array}{l}\text { Available resource management, Work plan, } \\
\text { Work order, Work performance }\end{array}$ & {$[2]$} \\
\hline & $\begin{array}{l}\text { Logistics service } \\
\text { improvement }\end{array}$ & $\begin{array}{l}\text { Information Quality, Interaction quality, Spell } \\
\text { effectiveness, Order accuracy, } \\
\text { Product status, Timeliness, Support system }\end{array}$ & [3] \\
\hline & $\begin{array}{l}\text { Logistics } \\
\text { operation } \\
\text { management } \\
\text { improvement }\end{array}$ & $\begin{array}{l}\text { Cost level improvement through unit price } \\
\text { management, } \\
\text { Improvement of planned logistics function through } \\
\text { order management, } \\
\text { Improved prevention of business confusion due to } \\
\text { unified order, } \\
\text { Improving work efficiency by improving procedures }\end{array}$ & {$[4]$} \\
\hline \multicolumn{2}{|c|}{ Logistics business support } & $\begin{array}{l}\text { Logistics management analysis, Logistics cost } \\
\text { management, } \\
\text { Logistics budget management, Logistics management } \\
\text { plan establishment work }\end{array}$ & {$[5]$} \\
\hline \multicolumn{2}{|c|}{ Business performance } & $\begin{array}{l}\text { Delivery-based yield accuracy, } \\
\text { Reduce lead time, Logistics cost reduction }\end{array}$ & [6] \\
\hline
\end{tabular}

\section{Results and Discussion}

In the study, analysis of the impact of SME logistics consulting on management performance is to test the control effect of logistics business support. To evaluate reliability and validity, Cronbach's Alpha coefficient is used for factor analysis and reliability analysis. To test the results, standard deviation and the mean were calculated using descriptive statistics, and Linear regression analysis was conducted for hypothesis testing

\section{3-1. Factor analysis of sample}

This study is a factor analysis which was conduct to test the validity of the sample surveyed on SMEs. Principal component analysis was used in order to simplify the factor loading value, and the factor loading value was expressed very high by more than 0.5 as the result of the analysis by adopting the orthogonal transformation method (Varimax) with Kaiser normalization. In addition, the result of factor analysis and exploratory factor analysis on the effect of logistics consulting of SMEs on business performance shows as given below, 4 questions, 4 Logistics service improvement, 4 Logistics operation management improvement, 4 logistics business support, 3 business results closely used for the analysis. [Table 2]. 
Table 2. Factor analysis

\begin{tabular}{|c|c|c|c|c|c|c|}
\hline \multirow{2}{*}{\multicolumn{2}{|c|}{ question }} & \multicolumn{5}{|c|}{ ingredient } \\
\hline & & \multirow{2}{*}{$\begin{array}{l}1 \\
.921 \\
\end{array}$} & \multirow{2}{*}{$\begin{array}{l}2 \\
.095\end{array}$} & \multirow{2}{*}{3} & \multirow{2}{*}{$\begin{array}{ll}4 \\
.128\end{array}$} & \multirow{2}{*}{$\begin{array}{l}5 \\
.128\end{array}$} \\
\hline \multirow{4}{*}{$\begin{array}{l}\text { Logistics } \\
\text { business } \\
\text { process }\end{array}$} & Improve order accuracy & & & & & \\
\hline & Product condition improvement & .911 & .095 & .176 & .130 & .156 \\
\hline & Timeliness improvement & .908 & .118 & .185 & .086 & .136 \\
\hline & Support system improvement & .890 & .055 & .150 & .093 & .200 \\
\hline \multirow{4}{*}{$\begin{array}{l}\text { Logistics } \\
\text { service } \\
\text { improvement }\end{array}$} & $\begin{array}{l}\text { Due to unit price management } \\
\text { Improvement of logistics planning } \\
\text { function }\end{array}$ & .072 & .916 & .124 & .180 & .074 \\
\hline & $\begin{array}{l}\text { Due to order management } \\
\text { Improvement of planned logistics } \\
\text { function }\end{array}$ & .116 & .895 & .196 & .129 & .081 \\
\hline & $\begin{array}{l}\text { Due to unified order } \\
\text { Improvement of prevention of } \\
\text { business confusion }\end{array}$ & .090 & .885 & .100 & .118 & .127 \\
\hline & $\begin{array}{l}\text { By improving the procedure } \\
\text { Improve work efficiency }\end{array}$ & .070 & .874 & .198 & .162 & .066 \\
\hline \multirow{4}{*}{$\begin{array}{l}\text { Logistics } \\
\text { business } \\
\text { support }\end{array}$} & Logistics accounting related work & .142 & .186 & .906 & .086 & .144 \\
\hline & Logistics management analysis work & .179 & .158 & .898 & .105 & .152 \\
\hline & Logistics cost management work & .164 & .198 & .897 & .110 & .109 \\
\hline & Logistics budget management & .224 & .097 & .782 & .115 & .108 \\
\hline \multirow{4}{*}{$\begin{array}{l}\text { Logistics } \\
\text { operation } \\
\text { management } \\
\text { improvement }\end{array}$} & Manage available resources & .133 & .083 & .124 & .897 & .151 \\
\hline & Work plan & .073 & .127 & .093 & .890 & .110 \\
\hline & Work order & .099 & .217 & .139 & .858 & .171 \\
\hline & Work performance & .121 & .182 & .054 & .814 & .247 \\
\hline \multirow{3}{*}{$\begin{array}{l}\text { Business } \\
\text { performance }\end{array}$} & Delivery compliance rate accuracy & .169 & .100 & .191 & .196 & .879 \\
\hline & Shortened lead time & .206 & .158 & .155 & .224 & .874 \\
\hline & Logistics cost reduction & .215 & .078 & .137 & .231 & .864 \\
\hline \multicolumn{2}{|l|}{ Dispersion \% } & 19.048 & 18.284 & 17.901 & 17.487 & 13.717 \\
\hline \multicolumn{2}{|l|}{ Accumulate \% } & 19.048 & 37.331 & 55.232 & 72.719 & 86.436 \\
\hline
\end{tabular}

\section{3-2. Reliability analysis}

This study analyzed the effect of logistics consulting of SMEs on business performance. Descriptive statistics and reliability analysis results, Entrepreneurial competence 0.8, Managerial ability 0.9, Technical competence 0.9, Social competence 0.95, Startup education 0.92, Technology start-up performance (Financial) 0.79, Technology start-up performance (Non-financial) 0.78 etc. All categories were analyzed to be satisfied with the reliability of 0.70 or higher. [Table 3].

Table 3. Reliability analysis

\begin{tabular}{|l|l|l|l|}
\hline Variable & Cronbach's Alpha & Average & Standard Deviation \\
\hline Logistics operation management & .686 & 3.771 & .579 \\
\hline Logistics business support & .690 & 3.801 & .558 \\
\hline Logistics service & .706 & 3.822 & .602 \\
\hline Logistics business process & .676 & 4.007 & .594 \\
\hline Business performance & .662 & 4.089 & .573 \\
\hline
\end{tabular}

\section{3-3. Correlation analysis}

In the study, the correlation analysis between variables was conducted in analyzing the impact of logistics consulting of SMEs on the business performance.

First, according to the table below, the correlation between logistics business process and logistics service recorded 0.289, Logistics operation management recorded 0.360, Logistics business support recorded 0.293 , Business performance recorded 0.456 .

Second, the correlation between logistics service and logistics operation management recorded 0.247 , 
Logistics business support recorded 0.417, Business performance recorded 0.421 .

Third, the correlation between logistics operation management and logistics support recorded 0.374, Business performance recorded 0.287 .

Fourth, the correlation between logistics support and business performance recorded 0.379 .

The correlation coefficient between the logistics business process and business performance recorded highest level. [Table 4].

Table 4. Correlation analysis

\begin{tabular}{|c|c|c|c|c|c|c|}
\hline \multicolumn{2}{|l|}{ Variable } & $\begin{array}{l}\text { logistics } \\
\text { Business } \\
\text { process }\end{array}$ & $\begin{array}{l}\text { Logistics } \\
\text { service }\end{array}$ & $\begin{array}{l}\text { Logistics } \\
\text { operation } \\
\text { management }\end{array}$ & $\begin{array}{l}\text { Logistics } \\
\text { business } \\
\text { support } \\
\end{array}$ & $\begin{array}{l}\text { Business } \\
\text { performance }\end{array}$ \\
\hline $\begin{array}{l}\text { logistics } \\
\text { Business } \\
\text { process } \\
\end{array}$ & Pearson & 1 & $.289^{* *}$ & $.360^{* *}$ & $.293^{* *}$ & $.456^{* *}$ \\
\hline $\begin{array}{l}\text { Logistics } \\
\text { service }\end{array}$ & Pearson & $.289^{* *}$ & 1 & $.247^{* *}$ & $.417^{* *}$ & $.421^{* *}$ \\
\hline $\begin{array}{l}\text { Logistics } \\
\text { operation } \\
\text { management }\end{array}$ & Pearson & $.360^{* *}$ & $.247^{* *}$ & 1 & $.374^{* *}$ & $.287^{* *}$ \\
\hline $\begin{array}{l}\text { Logistics } \\
\text { business } \\
\text { support }\end{array}$ & Pearson & $.293^{* *}$ & $.417^{* *}$ & $.374^{* *}$ & 1 & $.379^{* *}$ \\
\hline \multirow{2}{*}{$\begin{array}{l}\text { Business } \\
\text { performance }\end{array}$} & Pearson & $.456^{* *}$ & $.421^{* *}$ & $.287^{* *}$ & $.379^{* *}$ & 1 \\
\hline & $\mathrm{N}$ & 150 & 150 & 150 & 150 & 150 \\
\hline
\end{tabular}

**. The correlation coefficient is significant at the 0.01 level (Both sides).

\section{3-4. Hypothesis test}

In this study, the effect of logistics consulting of SMEs on business performance was analyzed. Through regression analysis, the influence and moderating effect between variables were analyzed. Analysis method is a kind of independent variable that systematically changes between the independent variable and the dependent variable. In the relationship between the dependent variable and the independent variable, the test whether the control variable has a moderating effect was divided into 3 phases.

Phase 1 is the regression analysis between independent and dependent variables. Phase 2 is the regression analysis between independent variable, control variable and dependent variables. Phase 3 is the conclusion which was drawn by regression analysis between the independent variable, the control variable, the interaction term (independent variable * control variable) and the dependent variable. According to the 1st, 2nd and 3rd phase of the moderation effect analysis, when the last three- phase interaction term (independent variable ${ }^{*}$ control variable) is add to the regression equation. If the explanatory power (R squared) is significantly increased under the significance level, it means that the explanatory power has a moderating effect.

H1. Improvement of logistics business process of logistics consulting, Logistics service improvement, Analysis of the impact of logistics operation management improvement on business performance R2: 0.306, F: 28.968 Improvement of logistics business process (.335), Logistics service improvement (.302), Logistics operation management improvement (.327) It was analyzed that it had a positive $(+)$ effect on business performance. [Table 5].

Table 5. Improvement of work process, Service improvement, Test the impact of operational management improvement on business performance

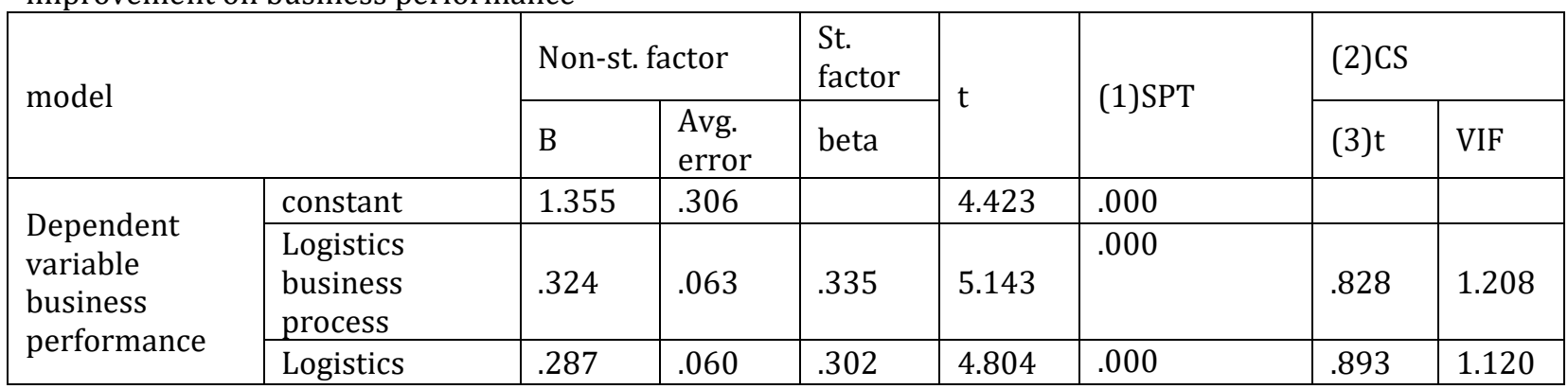




\begin{tabular}{|l|l|l|l|l|l|l|l|l|}
\hline & service & & & & & & & \\
\cline { 2 - 8 } & $\begin{array}{l}\text { Logistics } \\
\text { operation } \\
\text { management }\end{array}$ & .314 & .064 & .327 & 4.877 & .000 & .848 & 1.179 \\
\hline $\begin{array}{l}\text { R : } .553 \\
\text { R Squared }: .306\end{array}$
\end{tabular}

(1) SPT: Significance probability tolerance

(2) CS: Collinearity statistics

(3) t: tolerance

H2. Analysis of the impact of accounting support on business performance R2: 0.143, F: 33.299 Logistics business support (.379), and it was analyze to have a positive (+) effect on business performance. [Table 6]

Table 6. Assessment of the impact of accounting support on business performance

\begin{tabular}{|c|c|c|c|c|c|c|c|c|}
\hline \multirow{2}{*}{\multicolumn{2}{|c|}{ model }} & \multicolumn{2}{|c|}{ Non-st. factor } & \multirow{2}{*}{$\begin{array}{l}\begin{array}{l}\text { St. } \\
\text { factor }\end{array} \\
\text { beta }\end{array}$} & \multirow{2}{*}{$\mathrm{t}$} & \multirow{2}{*}{ SPT (1) } & \multicolumn{2}{|l|}{$\operatorname{CS}(2)$} \\
\hline & & B & $\begin{array}{l}\text { Avg. } \\
\text { error }\end{array}$ & & & & tolerance & VIF \\
\hline \multirow{2}{*}{$\begin{array}{l}\text { Dependent } \\
\text { variable } \\
\text { business } \\
\text { performance } \\
\end{array}$} & constant & 2.613 & .259 & & 10.102 & .000 & & \\
\hline & $\begin{array}{l}\text { Logistics } \\
\text { business } \\
\text { support }\end{array}$ & .389 & .067 & .379 & 5.771 & .000 & 1.000 & 1.000 \\
\hline \multicolumn{4}{|c|}{$\begin{array}{l}\text { R: .379a } \\
\text { R Squared: .143 }\end{array}$} & \multicolumn{5}{|c|}{$\begin{array}{l}\text { Mean square: } 9.421 \\
\text { F: } 33.299\end{array}$} \\
\hline
\end{tabular}

(1) SPT: Significance probability tolerance

(2) CS: Collinearity statistics

H3-1 Analysis of the moderating effect of logistics business support in the impact of business process on business performance. With effect of control in assisting business in logistics, a few things are found that phase 1 represents R2:0.208 and significance probability:0000, phase 2 shows R2:0.273 and significance probability:0000 and phase 3 represents R2: 0.298 and significance probability:0000. Actually, it is found that the value of R2 has gradually increased and the level of significance has been higher than $p<0.05$, which means that business in logistics is expected to improve performance. [Table 7].

Table 7. Analysis of the moderating effect of logistics business support in the impact of business process on business performance

\begin{tabular}{|c|c|c|c|c|c|c|c|c|c|c|}
\hline \multirow{2}{*}{ step } & \multirow{2}{*}{$\begin{array}{l}\text { Independen } \\
\text { t variable }\end{array}$} & \multicolumn{2}{|c|}{ (1)Non-st. } & \multirow{2}{*}{$\begin{array}{l}\text { (2)St. } \\
\text { beta }\end{array}$} & \multirow{2}{*}{$\mathrm{t}$} & \multirow{2}{*}{$\begin{array}{l}(3) \\
\text { SPT }\end{array}$} & \multirow{2}{*}{$\begin{array}{l}(4) \\
\text { R1 }\end{array}$} & \multirow{2}{*}{$\mathrm{F}$} & \multicolumn{2}{|c|}{$\begin{array}{ll}\text { Change } & \text { in } \\
\text { statistics } & \end{array}$} \\
\hline & & B & $\begin{array}{l}\text { Avg. } \\
\text { error }\end{array}$ & & & & & & $\begin{array}{l}(5) \\
\text { R2 }\end{array}$ & $\begin{array}{l}\mathrm{F} \\
\text { Change }\end{array}$ \\
\hline \multirow[b]{2}{*}{1} & constant & 2.328 & .247 & 247 & 9.441 & .000 & \multirow[b]{2}{*}{208} & \multirow[b]{2}{*}{52.106} & \multirow[b]{2}{*}{.208} & \multirow[b]{2}{*}{52.106} \\
\hline & $\begin{array}{l}\text { Logistics } \\
\text { business } \\
\text { process }\end{array}$ & .439 & .061 & .061 & 7.218 & .000 & & & & \\
\hline \multirow{3}{*}{2} & constant & 1.585 & .295 & .295 & 5.380 & .000 & \multirow{3}{*}{.273} & \multirow{3}{*}{37.238} & \multirow{3}{*}{.066} & \multirow{3}{*}{17.935} \\
\hline & $\begin{array}{l}\text { Logistics } \\
\text { business } \\
\text { process }\end{array}$ & .364 & .061 & .061 & 5.951 & .000 & & & & \\
\hline & $\begin{array}{l}\text { logistics } \\
\text { Business } \\
\text { support }\end{array}$ & .275 & .065 & .065 & 4.235 & .000 & & & & \\
\hline \multirow{4}{*}{3} & constant & 1.020 & 1.032 & 1.032 & .988 & .324 & \multirow{4}{*}{.298} & \multirow{4}{*}{27.874} & \multirow{4}{*}{.025} & \multirow{4}{*}{6.919} \\
\hline & $\begin{array}{l}\text { Logistics } \\
\text { business } \\
\text { process }\end{array}$ & 1.034 & .262 & .262 & 3.948 & .000 & & & & \\
\hline & $\begin{array}{l}\text { logistics } \\
\text { Business } \\
\text { support }\end{array}$ & 1.007 & .285 & .285 & 3.528 & .001 & & & & \\
\hline & Interaction & .187 & .071 & .071 & 2.630 & .009 & & & & \\
\hline
\end{tabular}


Dependent variable: business performance

(1) Non-st:: Non-standardization Coefficient

(2) St:: standardization Coefficient

(3) SPT: Significance probability tolerance

$\begin{array}{ll}\text { (4) R1: R Squared } & \text { (5) R2: Squared change }\end{array}$

H3-2. Analysis of the moderating effect of logistics service support in the impact of logistics service on business performance. With effect of control in assisting business in logistics, a few things are found that phase 1 represents R2:0.177 and significance probability:0.000, phase 2 shows R2:0.227 and significance probability:0.000 and phase 3 represents R2: 0.278 and significance probability:0000. Actually, it is found that the value of R2 has gradually increased and the level of significance has been higher than $\mathrm{p}<0.05$, which means that logistics service is expected to improve performance. [Table 8].

Table 8. Analysis of the moderating effect of logistics service support in the impact of logistics service on business performance

\begin{tabular}{|c|c|c|c|c|c|c|c|c|c|c|}
\hline \multirow{2}{*}{ step } & \multirow{2}{*}{$\begin{array}{l}\text { Independen } \\
\text { t variable }\end{array}$} & \multicolumn{2}{|c|}{ (1)Non-st. } & \multirow{2}{*}{$\frac{(2) \mathrm{St} .}{\text { beta }}$} & \multirow{2}{*}{$\mathrm{t}$} & \multirow{2}{*}{$\begin{array}{l}(3) \\
\text { SPT }\end{array}$} & \multirow{2}{*}{$\begin{array}{l}(4) \\
\mathrm{R} 1\end{array}$} & \multirow{2}{*}{$\mathrm{F}$} & \multicolumn{2}{|c|}{$\begin{array}{ll}\text { Change } & \text { in } \\
\text { statistics } & \end{array}$} \\
\hline & & B & $\begin{array}{l}\text { Avg. } \\
\text { error }\end{array}$ & & & & & & $\begin{array}{l}5) \\
\text { R2 } \\
\end{array}$ & $\begin{array}{l}\mathrm{F} \\
\text { Change }\end{array}$ \\
\hline \multirow[b]{2}{*}{1} & constant & 2.559 & .237 & & 10.819 & .000 & \multirow[b]{2}{*}{.177} & \multirow[b]{2}{*}{42.929} & \multirow[b]{2}{*}{.177} & \multirow[b]{2}{*}{42.929} \\
\hline & $\begin{array}{l}\text { Logistics } \\
\text { service }\end{array}$ & .401 & .061 & .421 & 6.552 & .000 & & & & \\
\hline \multirow{3}{*}{2} & constant & 1.972 & .282 & & 6.987 & .000 & \multirow{3}{*}{.227} & \multirow{3}{*}{29.130} & \multirow{3}{*}{.050} & \multirow{3}{*}{12.787} \\
\hline & $\begin{array}{l}\text { Logistics } \\
\text { service }\end{array}$ & .303 & .065 & .319 & 4.640 & .000 & & & & \\
\hline & $\begin{array}{l}\text { Logistics } \\
\text { business } \\
\text { support } \\
\end{array}$ & .252 & .071 & .246 & 3.576 & .000 & & & & \\
\hline \multirow{4}{*}{3} & constant & 1,799 & 1.051 & & 1.712 & .088 & \multirow{4}{*}{25.284} & \multirow{4}{*}{25.284} & \multirow{4}{*}{.051} & \multirow{4}{*}{13.821} \\
\hline & $\begin{array}{l}\text { Logistics } \\
\text { service }\end{array}$ & 1.347 & .288 & 1.416 & 4.680 & .000 & & & & \\
\hline & $\begin{array}{l}\text { Logistics } \\
\text { business } \\
\text { support }\end{array}$ & 1.271 & .283 & 1.239 & 4.500 & .000 & & & & \\
\hline & Interaction & .279 & .075 & 1.775 & 3.718 & .000 & & & & \\
\hline
\end{tabular}

Dependent variable: business performance

(1) Non-st:: Non-standardization Coefficient

(3) SPT: Significance probability tolerance

(2) St:: standardization Coefficient

(5) R2: Squared change

H3-3. Analysis of the moderating effect of logistics business support in the impact of logistics operation management on business performance. With effect of control in assisting business in logistics, a few things are found that phase 1 represents R2:0.107 and significance probability:0.000, phase 2 shows R2:0.192 and significance probability:0.000 and phase 3 represents R2: 0.267 and significance probability:0000. Actually, it is found that the value of R2 has gradually increased and the level of significance has been higher than $\mathrm{p}<0.05$, which means that logistics operation management is expected to improve performance. [Table 9].

Table 9. Analysis of the moderating effect of logistics business support in the impact of logistics operation management on business performance

\begin{tabular}{|c|c|c|c|c|c|c|c|c|c|c|}
\hline \multirow{2}{*}{ step } & \multirow{2}{*}{$\begin{array}{l}\text { Independent } \\
\text { variable }\end{array}$} & \multicolumn{2}{|c|}{ (1)Non-st. } & \multirow{2}{*}{$\frac{(2) \mathrm{St} .}{\text { beta }}$} & \multirow{2}{*}{$\mathrm{t}$} & \multirow{2}{*}{$\begin{array}{l}(4) \\
\text { SPT }\end{array}$} & \multirow{2}{*}{$\begin{array}{l}(5) \\
\text { R1 }\end{array}$} & \multirow{2}{*}{$\mathrm{F}$} & \multicolumn{2}{|c|}{$\begin{array}{ll}\text { Change } & \text { in } \\
\text { statistics } & \end{array}$} \\
\hline & & B & $\begin{array}{l}\text { (3) } \\
\text { Avg. }\end{array}$ & & & & & & $\begin{array}{l}\text { (6) } \\
\text { R2 }\end{array}$ & $\begin{array}{l}\mathrm{F} \\
\text { Change }\end{array}$ \\
\hline \multirow[b]{2}{*}{1} & constant & 2.880 & .251 & & 11.482 & .000 & \multirow[b]{2}{*}{.107} & \multirow[b]{2}{*}{23.789} & \multirow[b]{2}{*}{$.107 a$} & \multirow[b]{2}{*}{23.789} \\
\hline & $\begin{array}{l}\text { Logistics } \\
\text { operation } \\
\text { management }\end{array}$ & .314 & .064 & .327 & 4.877 & .000 & & & & \\
\hline \multirow{2}{*}{2} & constant & 2.039 & .302 & & 6.745 & .000 & \multirow{2}{*}{.192} & \multirow{2}{*}{23.463} & \multirow{2}{*}{$.085 b$} & \multirow{2}{*}{20.774} \\
\hline & Logistics & .222 & .065 & .231 & 3.438 & .000 & & & & \\
\hline
\end{tabular}




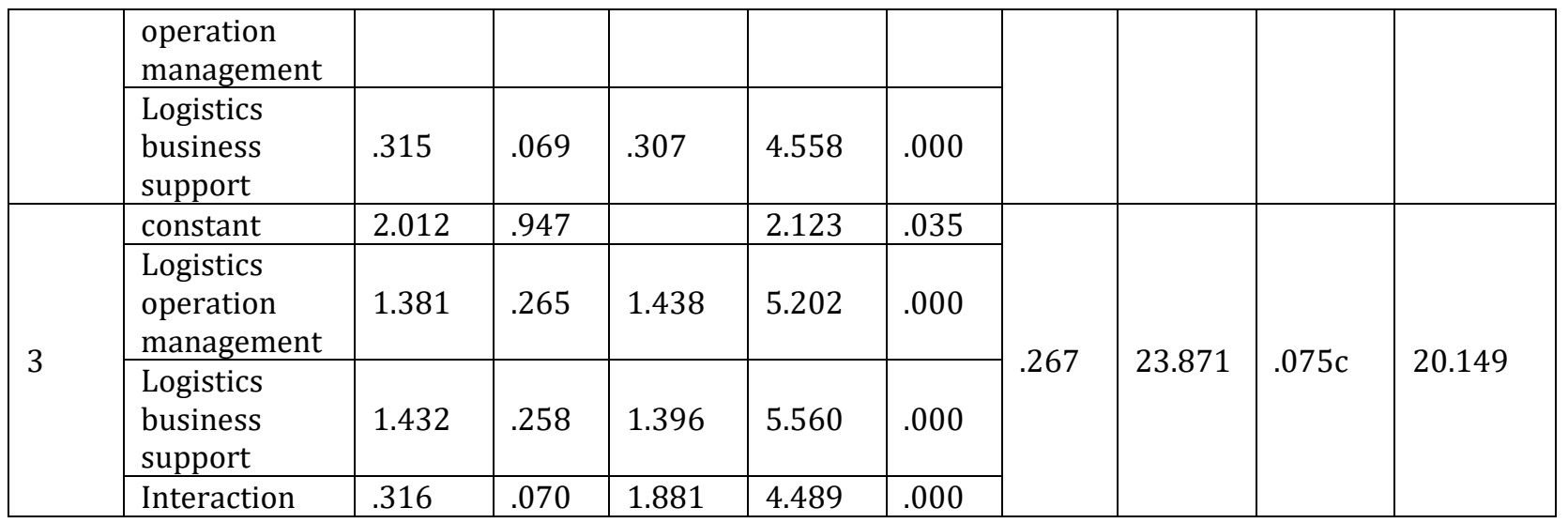

Dependent variable: business performance

(1) Non-st:: Non-standardization Coefficient

(3) Avg.: Average error

(2) St:: standardization Coefficient

(5) R1: R Squared

(4) SPT: Significance probability tolerance

(6) R2: Squared change

\section{Conclusions}

In this study, logistics consulting is being promoted for the self-sustainability of SMEs. To analyze the business performance of SMEs through logistics consulting, we conducted online and offline surveys of 150 SMEs. In addition, we tried to analyze how much the logistics consulting of SMEs affects business performance. As a measurement independent variable, improvement of logistics work process, improvement of logistics service, and improvement of logistics operation management are set up, and the control variable was tested for the influence of logistics business support, and the dependent variable was tested business performance as a variable. Logistics consulting of SMEs was analyzed to have a significant impact in the business performance of logistics business process improvement, logistics service improvement, and logistics operation management improvement, in terms of the impact of improving logistics business processes, improving logistics services, and improving logistics operation management. It was also analyzed that logistics support has a moderating effect in improving business performance. In order to increase the self-sustainability of SMEs, it is important to improve logistics business processes, logistics services, and logistics operation management. As a way to increase self-sustainability, logistics consulting should continue to support logistics business processes, logistics services, logistics operation management and logistics business support.

\section{Acknowledgements}

This research was financially supported by Hansung University.

\section{References}

[1] Joo hyun-Eom (2017). Share of SMEs in the domestic logistics industry "99.9\%", Cargo news. http://www.cargonews.co.kr/news/articleView.html?idxno=36250

[2] Jeong sik-Park (2006). A study of structural relationship between logistics service quality, customer satisfaction, customer loyalty in internet shopping malls. Myong ji University Graduate School http://www.riss.kr/search/detail/DetailView.do?p_mat_type=be54d9b8bc7cdb09\&control_no=5de8 1cae703c59bcffe0bdc3ef48d419

[3] Hyo seop-Lee (2017). The effect of logistics information system introduction factor on logistics performance: focused on the moderating effect of business strategy and ISP suitability http://www.riss.kr/search/detail/DetailView.do?p_mat_type=be54d9b8bc7cdb09\&control_no=dfac3 1f4f0e55788ffe0bdc3ef48d419

[4] Young ho-Lee (2016). Impact of SME consulting process and consulting environment on consulting performance Doctoral dissertation. Kyung il University Graduate School http://www.riss.kr/search/detail/DetailView.do?p_mat_type=be54d9b8bc7cdb09\&control_no=adb1 35ee9d29605dffe0bdc3ef48d419

[5] Doo hyun-Park (2019). A Study on the effect of Management Consulting Service Quality on Customer Satisfaction and Performance : for customers who use management consulting company

Master's thesis. Gyeong gi University Graduate School. 
http://www.riss.kr/search/detail/DetailView.do?p_mat_type=be54d9b8bc7cdb09\&control_no=e770d eda00ef9220ffe0bdc3ef48d419

[6] Beo Deul-Gang(2005). Relative differences in service quality in the knowledge-based industry : an empirical study of consulting. Master's thesis. Seoul National University Graduate School http://www.riss.kr/search/detail/DetailView.do?p_mat_type=be54d9b8bc7cdb09\&control_no=9ed99 2bdcda0583c

[7] Hong ju-Kwak (2008). A Study on the Factors for Improving Performance in Management Consulting and Real Estate Consulting Doctoral dissertation. Gyeong gi University Graduate School http://www.riss.kr/search/detail/DetailView.do?p_mat_type=be54d9b8bc7cdb09\&control_no=9b65 a8a8f3380b93ffe0bdc3ef48d419 [9] Tse, D. K. \& Peter, C. W. (1988) Models of Consumer Satisfaction: An Extension. Journal of Marketing Research, May,204-212.

[8] Dong jun-Lee (2016). A study on standardization of operation process for 3rd party logistics company distribution center.

Master's thesis. Seo kyeong University Graduate School. http://www.riss.kr/search/detail/DetailView.do?p_mat_type=be54d9b8bc7cdb09\&control_no=97c2 85110aa55565ffe0bdc3ef48d419 [11] Sirdeshmukh, D., Singh, J., \& Sabol, B. (2002) Consumer Trust, Value and Loyalty in Relational Exchanges. Journal of Marketing,66(1),15-37.

[9] Lee Sook-Park (2008). The effects of logistics service quality and relationship orientation of 3PL firms on supply chain performance Doctoral dissertation. Chon nam National University Graduate School. http://www.riss.kr/search/detail/DetailView.do?p_mat_type=be54d9b8bc7cdb09\&control_no=6d2e c50ab77bb80effe0bdc3ef48d419 [13] Rynning,M. (1992) Successful consulting with small and medium-sized vs large clients:meeting the needs of the client?.International Business Journal,11(1), pp. 47-60.

[10] Chang deok-Kim (2008). A Study on the Actual Conditions of Logistics Cost Management and Improvement Plan of Korean Companies.

Master's thesis. Changwon University Graduate School http://www.riss.kr/search/detail/DetailView.do?p_mat_type=be54d9b8bc7cdb09\&control_no=a44fd 44c3b2874c9ffe0bdc3ef48d419

[11] Deok hee-Lee (2009). A study on the impact of management consulting service quality on customer loyalty.

Master's thesis, Graduate School, Changwon University.

http://www.riss.kr/search/detail/DetailView.do?p_mat_type=be54d9b8bc7cdb09\&con rol_no=d5a93930dc39b9dfffe0bdc3ef48d419

[12] Byeong sam-Park (2007). A study on the implementation of production planning system for real time capable to promise in supply chain management: concentrated on the practice of W. company Master's thesis. Changwon University Graduate School.

http://www.riss.kr/search/detail/DetailView.do?p_mat_type=be54d9b8bc7cdb09\&control_no=b 687b4e4c5a65688ffe0bdc3ef48d419 
